This item was submitted to Loughborough's Research Repository by the author.

Items in Figshare are protected by copyright, with all rights reserved, unless otherwise indicated.

\title{
A comparison between guidance laws for AUVs using relative kinematics
}

PLEASE CITE THE PUBLISHED VERSION

https://doi.org/10.1109/oceanse.2017.8084781

PUBLISHER

IEEE

VERSION

AM (Accepted Manuscript)

\section{PUBLISHER STATEMENT}

(c) 2017 IEEE. Personal use of this material is permitted. Permission from IEEE must be obtained for all other uses, in any current or future media, including reprinting/republishing this material for advertising or promotional purposes, creating new collective works, for resale or redistribution to servers or lists, or reuse of any copyrighted component of this work in other works.

\section{LICENCE}

All Rights Reserved

\section{REPOSITORY RECORD}

Abudureheman, Bilale, A Savvaris, and A Tsourdos. 2017. "A Comparison Between Guidance Laws for Auvs Using Relative Kinematics”. Loughborough University. https://hdl.handle.net/2134/14268866.v1. 


\title{
A Comparison between Guidance Laws for AUVs using Relative Kinematics
}

\author{
Bilal Abdurahman, A. Savvaris, A. Tsourdos \\ School of Aerospace, Transport and Manufacturing \\ Cranfield University \\ Bedfordshire, UK \\ a.bilale@cranfield.ac.uk
}

\begin{abstract}
This paper presents a comparison between three popular guidance laws for path following of autonomous underwater vehicles: switching enclosure-based Line-Of-Sight (LOS), lookahead-based LOS, and vector field guidance laws. The equations of motion employ the concept of the relative kinematics, and a nonlinear controller is applied together with the guidance systems during path-following. The optimal tuning values for each guidance are selected using the Pareto efficiencies from multiple simulations in terms of providing low cross-track error and control effort. Performance analysis are carried out for a waypoint following scenario both with and without significant constant and irrotational ocean currents as disturbances. Simulation results are also presented using the model of an AUV.
\end{abstract}

Keywords-line-of-sight; guidance laws; path-following control; current disturbance; underwater vehicles

\section{INTRODUCTION}

Underwater vehicle applications, e.g. inspection of pipelines and surveying require accurate and efficient execution of path-following tasks. This paper will illustrate the characteristics of and a comparison between three popular guidance laws: the Switching Enclosure-based Line-Of-Sight (SELOS), the Lookahead-based LOS (LLOS), and the Vector Field (VF) guidance laws [1-7]. Under ocean current disturbances, their performances will be poor if the drift caused by the current is not taken into account in the design, and the Integral LOS (ILOS) designs have been a common approach in dealing with ocean current disturbances, which can compensate for this drift [3], [6].

The LLOS guidance is perhaps the most popular scheme because it is easy and computationally simpler than the enclosure-based LOS (ELOS) and VF guidance. However, the ELOS scheme provides a variable lookahead distance $\Delta$, where this is fixed in the LLOS scheme, and this distinct feature could be attributed to why it performs better than the LLOS scheme (see carrot-chasing and nonlinear guidance law in [8]). In [8], VF guidance applied to Unmanned Air Vehicles (UAVs) was superior to the other two schemes above in terms of producing low cross-track error and requiring low control effort under wind disturbances. Nevertheless, VF guidance is known to exhibit chattering effect [8-9], and the comparison in [8] models the relative velocity differently. In [9], a comparison was made between an Integral LLOS (ILLOS) and VF guidance based on experimental results, where the VF guidance performed slightly better, but again with some chattering while the ILLOS scheme provided smoother servo signals.

The SELOS scheme is developed as a modification to the ELOS guidance to address the need for additional pathapproaching strategies required when the cross-track error becomes large. It is simpler and preserves the properties of the ELOS guidance unlike previously proposed modifications [1]. The guidance laws are implemented at both the kinetic and kinematic levels, and employ the relative kinematics in [1]. A nonlinear controller is applied during path-following. The comparison considers the underactuated case of an AUV's performance during waypoint following of straight-lines with and without current disturbances. Simulation and comparison results are provided at the end.

\section{SyStem MOdEL AND CONTROL OBJECTIVE}

\section{A. System Model}

The modified system model considers the horizontal dynamics of a class of underwater vehicles that can be described by the 3-DOF model [5]:

$$
\begin{gathered}
\dot{\boldsymbol{\eta}}=\boldsymbol{R}(\psi) \boldsymbol{v} \\
M \dot{\boldsymbol{v}}+\boldsymbol{C}(\boldsymbol{v}) \boldsymbol{v}+\boldsymbol{D}(\boldsymbol{v}) \boldsymbol{v}+\boldsymbol{g}(\boldsymbol{\eta})=\boldsymbol{B} \boldsymbol{f}
\end{gathered}
$$

where $\eta \triangleq[x, y, \psi]^{T}$ is the horizontal position and orientation of the vehicle in inertial frame $i$, and $v \triangleq[u, v, r]^{T}$ is the vector of absolute velocities of the vehicle in surge, sway, and yaw in body-fixed frame $b$, and $\boldsymbol{R}(\psi)$ is the transformation matrix from $b$ to $i$. Furthermore, $\boldsymbol{M}=\boldsymbol{M}^{T}>0$ is the system mass-inertia matrix including added mass, $\boldsymbol{C}$ is the Coriolis and centripetal matrix including rigid-body and added-mass terms, $\boldsymbol{D}>0$ is the damping matrix including linear and quadratic terms, $\boldsymbol{g}(\boldsymbol{\eta}) \triangleq\left[\begin{array}{lll}0 & 0 & 0\end{array}\right]^{T}$ is the gravitational and restoring forces and moments. The control input vector $\boldsymbol{M}^{-1} \boldsymbol{B} \boldsymbol{f} \triangleq\left[\tau_{u}, 0, \tau_{\psi}\right]^{T}$ consists of surge thrust and yaw moment, where $f \in \mathscr{R}^{2}$ is the actuator input vector and $\boldsymbol{B} \in \mathscr{R}^{3 \times 2}$ is the actuator configuration matrix. This structure of the control input vector is obtained by assuming that $b$ is 
positioned in the pivot point such that yaw moment has no effect on sway motion [10]. Note that system (2) is underactuated since the dimension of $f$ is less than that of the system.

According to [1], the relative kinematics define the velocities as follows. The relative velocities of the vehicle relative to $i$ frame $\boldsymbol{v}_{r}^{i}$ is

$$
\boldsymbol{v}_{r}^{i} \triangleq \boldsymbol{v}+\boldsymbol{v}_{c}=\left[u_{r}^{i}, v_{r}^{i}, r_{r}^{i}\right]^{T}
$$

where $\boldsymbol{v}_{c} \triangleq\left[u_{c}, v_{c}, 0\right]^{T}$ is the ocean current velocity in $b$. The relative velocities of the vehicle relative to FLOW frame $\boldsymbol{v}_{r}^{f}$ is:

$$
\boldsymbol{v}_{r}^{f} \triangleq \boldsymbol{v}-\boldsymbol{v}_{c}=\left[u_{r}^{f}, v_{r}^{f}, r_{r}^{f}\right]^{T}
$$

Current velocities $u_{c}$ and $v_{c}$ are given by: $u_{c}=$ $V_{c} \cos \left(\beta_{c}-\psi\right), v_{c}=V_{c} \sin \left(\beta_{c}-\psi\right)$, where $V_{c} \triangleq \sqrt{u_{c}^{2}+v_{c}^{2}}>$ 0 and $\beta_{c}=\operatorname{atan} 2\left(V_{y}, V_{x}\right)$ are the current intensity and heading in $i$, and $V_{c} \triangleq\left[V_{x}, V_{y}, 0\right]^{T}$ is the ocean current velocity in $i$. The ocean current is assumed constant (slowly-varying) and irrotational in $i$, which gives $\dot{\boldsymbol{V}}_{c}=\mathbf{0}$ and $\dot{\mathbf{v}}_{c}=$ $\left[r_{r}^{i} v_{c},-r_{r}^{i} u_{c}, 0\right]^{T}$. Substituting (3) and (4) into the kinetics and kinematics in (1) and (2), respectively, yields the relative equations of motion:

$$
\begin{gathered}
\dot{\eta}=R(\psi) v_{r}^{i} \\
M \dot{v}_{r}^{f}+C\left(v_{r}^{f}\right) v_{r}^{f}+D\left(v_{r}^{f}\right) v_{r}^{f}+g(\eta)=B f .
\end{gathered}
$$

Expanding (3) gives

$$
\begin{aligned}
& \dot{x}=u_{r}^{i} \cos \psi-v_{r}^{i} \sin \psi \\
& \dot{y}=u_{r}^{i} \sin \psi+v_{r}^{i} \cos \psi \\
& \dot{\psi}=r_{r}^{i}
\end{aligned}
$$

The system matrices have the following structure:

$$
\begin{gathered}
\boldsymbol{M} \triangleq\left[\begin{array}{ccc}
m_{11} & 0 & 0 \\
0 & m_{22} & m_{23} \\
0 & m_{23} & m_{33}
\end{array}\right] \\
\boldsymbol{C}\left(\boldsymbol{v}_{r}^{f}\right) \triangleq\left[\begin{array}{ccc}
0 & 0 & c_{13}\left(r_{r}^{f}, v_{r}^{f}\right) \\
0 & 0 & c_{23}\left(u_{r}^{f}\right) \\
-c_{13}\left(r_{r}^{f}, v_{r}^{f}\right) & -c_{23}\left(u_{r}^{f}\right) & 0
\end{array}\right], \\
\boldsymbol{D}\left(\boldsymbol{v}_{r}^{f}\right) \triangleq \operatorname{diag}\left\{d_{11}\left(u_{r}^{f}\right), d_{22}\left(v_{r}^{f}\right), d_{33}\left(r_{r}^{f}\right)\right\}
\end{gathered}
$$

The particular structure of the system matrices in (10) and (11) are obtained by assuming that the vehicle is symmetric in port-starboard, and that the body-fixed coordinate system is located along the centre-line of the vehicle [5]. The relative system dynamics are then obtained by expanding (6):

$$
\dot{u}_{r}^{f}=-\frac{1}{m_{11}}\left(c_{13} r_{r}^{f}+d_{11} u_{r}^{f}-\tau_{\mathrm{u}}\right)
$$

$$
\begin{gathered}
\dot{v}_{r}^{f}=-\frac{1}{m_{22}}\left(c_{23} r_{r}^{f}+d_{22} v_{r}^{f}\right)-m_{23} \dot{r}_{r}^{f}, \\
\dot{r}_{r}^{f}=\frac{1}{m_{33}}\left(c_{13} u_{r}^{f}+c_{23} v_{r}^{f}-d_{33} r_{r}^{f}+\tau_{\psi}\right)-m_{23} \dot{v}_{r}^{f} .
\end{gathered}
$$

where the arguments of the elements of $\boldsymbol{C}$ and $\boldsymbol{D}$ are omitted. Note that since the yaw component of the current $V_{\psi}=0$, the relative yaw rates are identical in both $i$ and FLOW frames, i.e. $r_{r}^{i}=r_{r}^{f}=r$.

\section{B. Control Objective}

The path-following problem considered is similar to a manoeuvring problem [4], which involves controlling the horizontal relative speed $U_{h r}^{i} \triangleq \sqrt{u_{r}^{i 2}+v_{r}^{i 2}}$ in $i$ of the vehicle towards the horizontal desired relative speed $U_{h d r}^{i} \geq V_{c}>0$ along the path, which is defined as:

$$
U_{h d r}^{i} \triangleq \sqrt{u_{d r}^{i 2}+v_{d r}^{i 2}} \triangleq \sqrt{\left(u_{d}+u_{c}\right)^{2}+\left(v_{d}+v_{c}\right)^{2}},
$$

where $u_{d r}^{i}$ and $v_{d r}^{i}$ are the desired relative surge and sway velocities in $i$, and $u_{d}$ and $v_{d}$ are the desired absolute velocities. Since sway DOF is not actuated, $v_{d}=0$, and thus from (16)

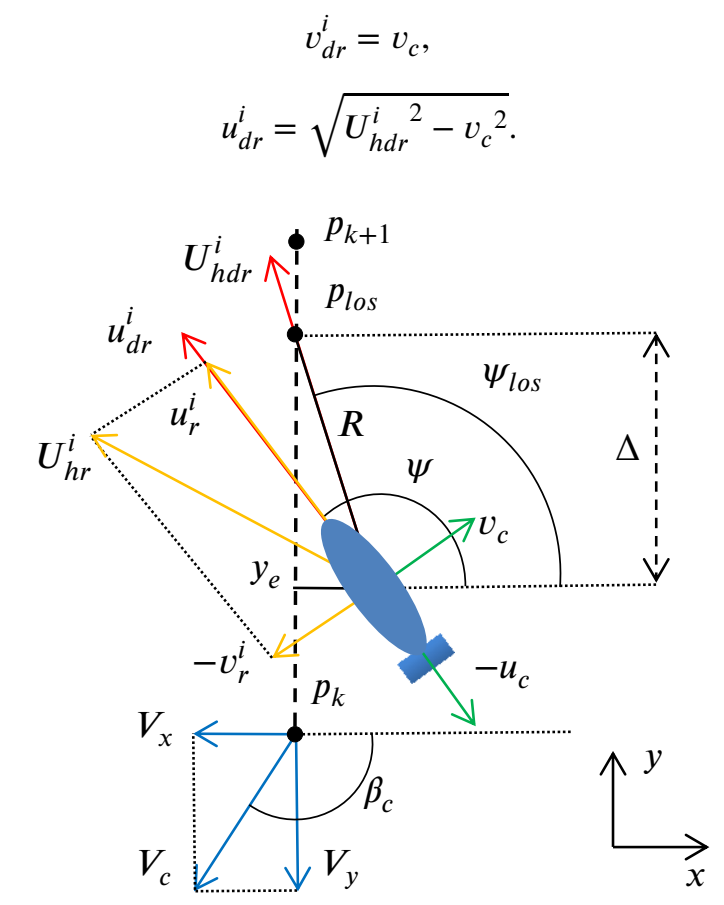

Figure 1. Geometry of ELOS guidance and velocity vectors

The control objectives are then reduced to controlling $u_{r}^{i}$ and $\psi$ such that:

$$
\begin{aligned}
& \lim _{t \rightarrow \infty} u_{r}^{i}(t)=u_{d r}^{i}(t), \\
& \lim _{t \rightarrow \infty} \psi(t)=\psi_{c r s}(t), \\
& \lim _{t \rightarrow \infty} y_{e}(t)=0 .
\end{aligned}
$$


where $\psi_{\text {crs }}$ is the desired course angle. The $i$-frame can be placed along the x-axis of the path-frame $P$ such that $y$ position of the vehicle becomes the cross-track error $y_{e}$.

Control of relative velocities instead of absolute velocities provides better energy efficiency since the hydrodynamic damping depends on $\boldsymbol{v}_{r}^{f}$ and the vehicle motion can benefit from the current velocities without actuator effort when $\beta_{c}$ coincides with the desired course angle.

\section{SElOs GUIDANCE}

The ELOS scheme employs a circle with radius $R$ around the vehicle to calculate the LOS angle $\psi_{\text {los }}$, which is found by solving the following equations online [4]:

$$
\begin{gathered}
\left(x_{l o s}-x\right)^{2}+\left(y_{l o s}-y\right)^{2}=R^{2}, \\
\frac{y_{l o s}-y_{k}}{x_{l o s}-x_{k}}=\frac{y_{k+1}-y_{k}}{x_{k+1}-x_{k}}=\alpha_{k}=\text { constant }, \\
\psi_{\text {los }} \triangleq \text { atan2 }\left(\frac{y_{l o s}-y}{x_{l o s}-x}\right) .
\end{gathered}
$$

where $\alpha_{k}$ is the path tangential angle. It is critical for $R$ to be greater than $\left|y_{e}\right|$, so that this circle-path interceptions exist, i.e. $R \geq\left|y_{e}\right|$ for all $y_{e}$. A large $R$ can guarantee $R \geq\left|y_{e}\right|$, but it will project a lookahead distance $\Delta$ too far ahead of the vehicle, resulting in a longer path convergence time. A smaller $R$ can decrease the path convergence time, but a pathapproaching strategy must be in place when $\left|y_{e}\right|>R$. $R$ must be sufficiently large so that solutions to (18) exist.

The SELOS scheme is a modification to ELOS which overcomes this drawback by employing a variable radius using a conditionally varying $R$, which is given by [1]:

$$
R=R_{\text {min }}+\frac{1}{2}\left[\frac{\left(a\left|y_{e}\right|-R_{\text {min }}\right)\left|y_{e}\right|-R_{\text {min }}}{c+|| y_{e}\left|-R_{\text {min }}\right|}+a\left|y_{e}\right|-R_{\text {min }}\right],
$$

where $a \geq 1$ is a design constant and $1 / c>0$ is the slope of the sigmoid function at the origin. For $a \approx 1$ and $c \approx 0$, (25) acts as a continuous switch such that:

$$
R \approx \begin{cases}R_{\text {min }} & \text { if }\left|y_{e}\right|<R_{\text {min }} \\ a\left|y_{e}\right| & \text { if }\left|y_{e}\right|>R_{\text {min }}\end{cases}
$$

which always guarantees solutions to (22) and hence, the existence of the circle-path interceptions.

\section{CONTROLler And CONVERGEnCE}

\section{A. Sideslip Compensation}

Aircrafts or marine crafts subject to winds or currents will experience lateral drift forces which will cause them to slide sideways, which can result in poor tracking if not taken into account in the design. Therefore, it is important to compensate for the sideslip when generating a desired heading angle. The sideslip angle $\beta$ is calculated using relative velocities in $i$ :

$$
\beta \triangleq \operatorname{atan} 2\left(\frac{v_{r}^{i}}{u_{r}^{i}}\right)
$$

The course angle $\psi_{\text {crs }}$ can be designed using the sideslip feedback (SF) to compensate for the drift, that is:

$$
\psi_{c r s} \triangleq \psi_{d}-\beta
$$

where $\psi_{d}$ is the desired heading given by the guidance law.

\section{B. Surge and Yaw Control}

The path-following controller is based on feedbacklinearization and sliding-mode control, which are given by:

$$
\begin{aligned}
\tau_{\psi}= & c_{13} u_{r}^{f}+c_{23} v_{r}^{f}-d_{33} r_{r}^{f} \\
& +m_{33}\left[\ddot{\psi}_{c r s}+\lambda\left(\dot{\psi}_{c r s}-r^{i}\right)+m_{23} \dot{v}_{r}^{f}+k_{\psi} \widetilde{\psi}+k_{d} s\right], \\
\tau_{u}= & c_{13} r_{r}^{f}+d_{11} u_{r}^{f}+m_{11}\left(\dot{u}_{d r}^{i}+k_{u} \tilde{u}_{r}^{i}\right) .
\end{aligned}
$$

where $k_{\psi}, k_{d}, k_{u}>0$ are constant gains and the error dynamics are defined as $\tilde{\psi} \triangleq \psi_{c r s}-\psi$ and $\tilde{u}_{r}^{i} \triangleq u_{d r}^{i}-u_{r}^{i}$. Stability and convergence analysis of the closed-loop error dynamics are provided in [1], which shows that the controller (29-30) satisfy the control objectives (19-21).

\section{PATH-Following PERFoRMANCE}

The path-following performances of these guidance laws for different tuning values are presented and analyzed in this section. Simulations were carried out using the model of Girona-500 AUV in waypoint following scenarios with and without current disturbance of $V_{c}=0.6 \mathrm{~m} / \mathrm{s}$ from Northeast direction, $\beta_{c}=\pi / 4$. The vehicle parameters are obtained from [11]. The initial conditions and desired states are set as $\boldsymbol{\eta}_{o}=[4,5,0]^{T}, \boldsymbol{v}_{r o}^{f}=\boldsymbol{v}_{r o}^{i}=[0,0,0]^{T}, U_{h d r}^{i}=1 \mathrm{~m} / \mathrm{s}$. The gains for the controller are as: $R_{k}=0.8, \lambda=120, k_{d}=25, k_{r}=$ $k_{a}=k_{\psi}=k_{1}=1, k_{u}=25$, where $R_{k}$ is the radius of acceptance for waypoint switching. The waypoints used are: $\quad w p x=\{10,14,21,26,32\}, w p y=\{5,22,22,8,17.5\}$. The control inputs saturate at $\pm 450 N$, and this is to make the simulation represent a more realistic vehicle behavior.

\section{A. SELOS Guidance}

The gains for SELOS guidance are as: $R_{\min }=0.9$, $a=1.001, c=0.001$. The path-following performance for

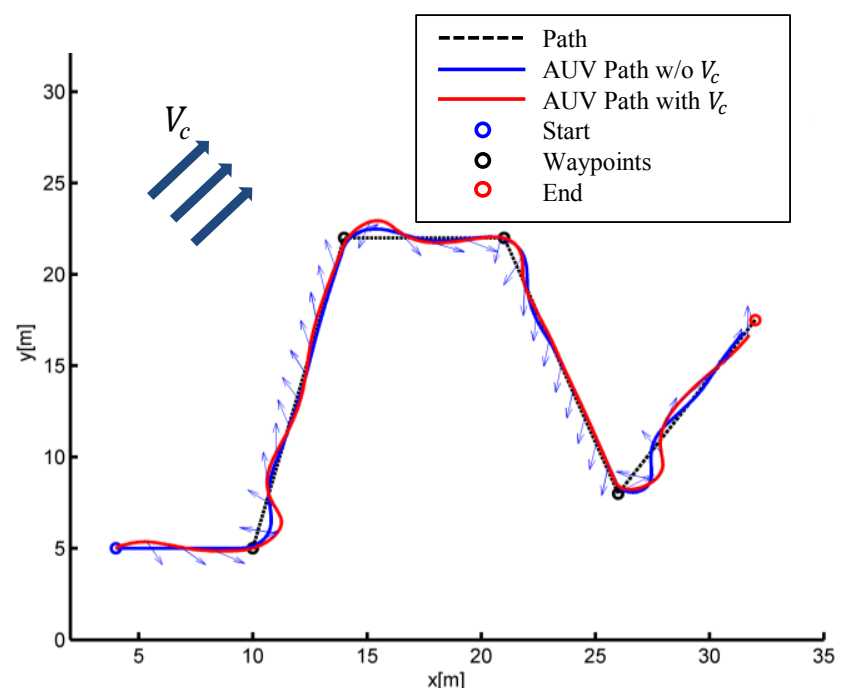

Fig. 2. Performance of SELOS guidance with $R_{\min }=0.9$. 
straight-line with and without current disturbance is shown in Fig. 2. It shows that the guidance system performs well in following the path with or without significant current disturbance. The Pareto efficiency plots with the cross-track error (CT) and the control effort (U) are shown in Figures 3 and 4 for both cases: with and without current disturbances.

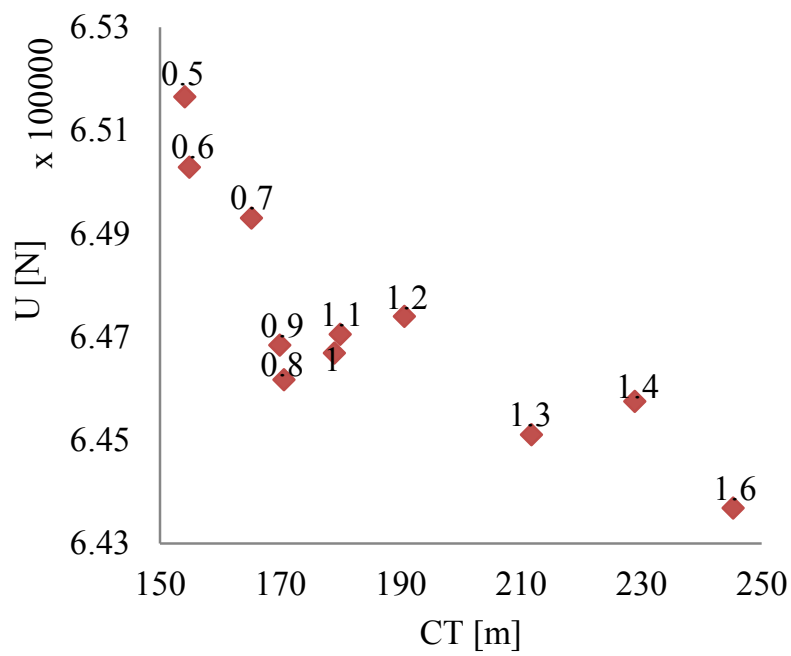

Fig. 3. Pareto efficiency of SELOS guidance without current disturbance.

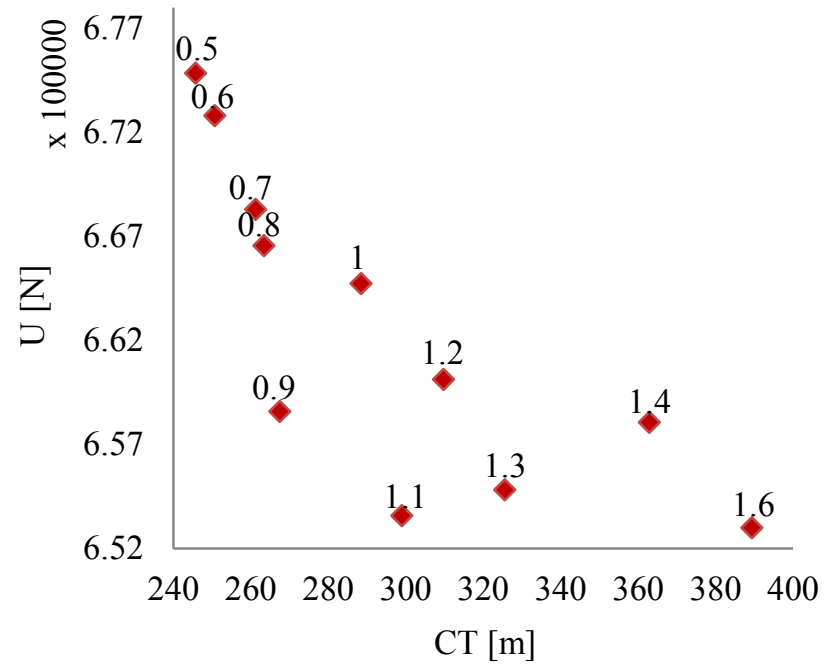

Fig. 4. Pareto efficiency of SELOS guidance with current disturbance of

$$
V_{c}=0.6 \mathrm{~m} / \mathrm{s} \text { and } \beta_{c}=\pi / 4 \text {. }
$$

\section{B. LLOS Guidance}

The desired headaing angle for the LLOS guidance law is given by [5] as

$$
\psi_{d}=\alpha_{k}-\tan ^{-1}\left(\frac{-y_{e}}{\Delta}\right)
$$

The path-following performance of LLOS guidance for waypoints consisting of straight-lines is shown in Fig. 5. It shows that the vehicle is able to follow the path accurately with and without a strong current disturbance. The pareto efficiency for the VF guidance is shown in Figures 6 and 7 also for both cases with and without current disturbance.

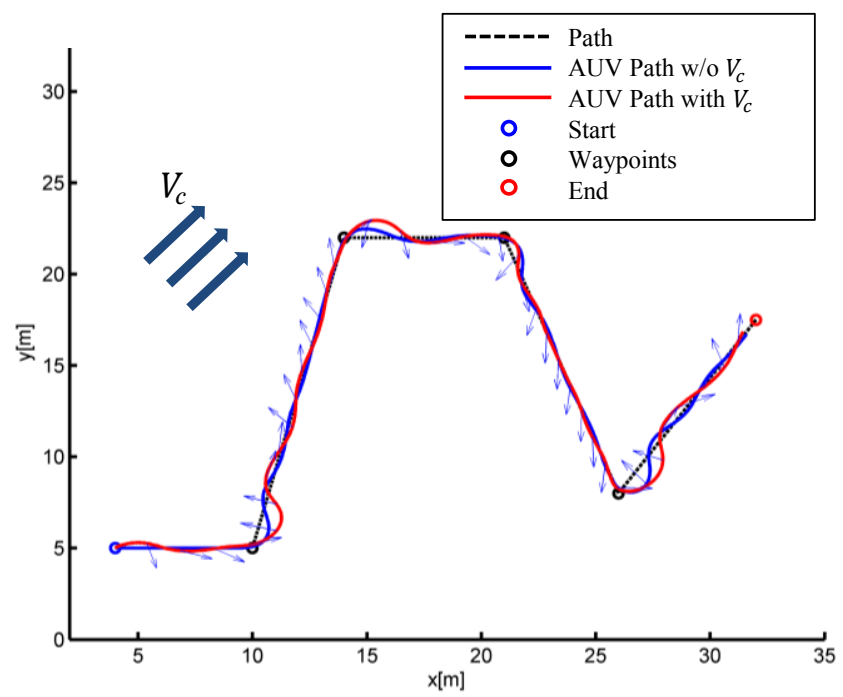

Fig. 5. Performance of SELOS guidance with $\Delta=0.4$.

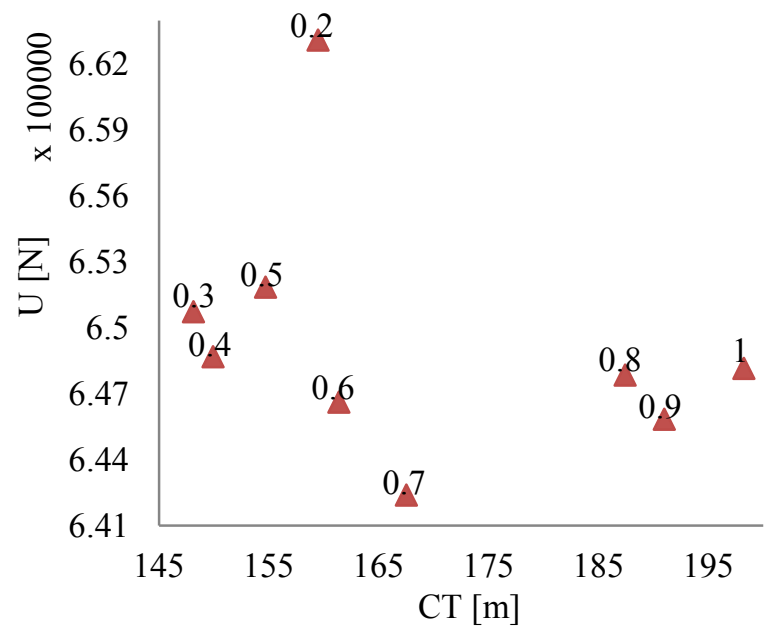

Fig. 6. Pareto efficiency of LLOS guidance without current disturbance.

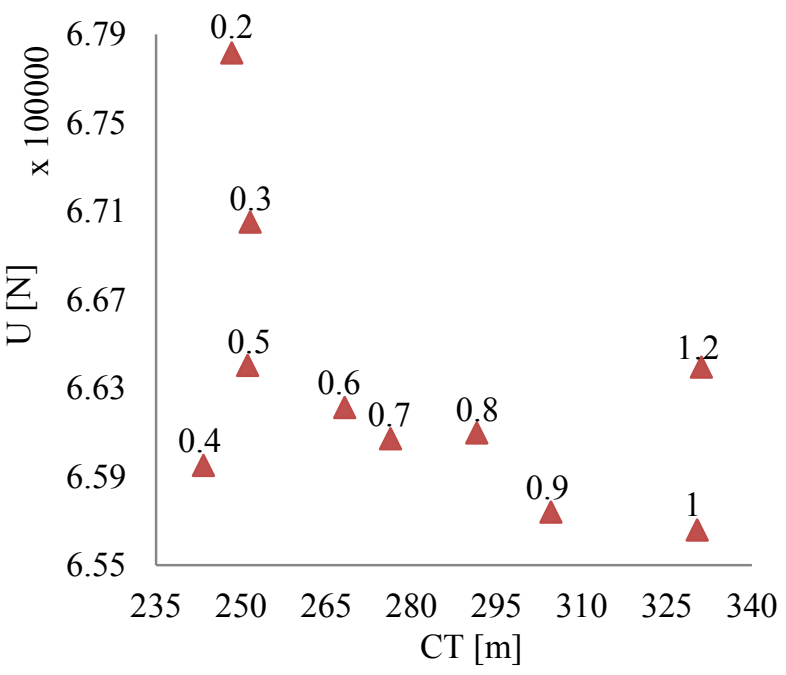

Fig. 7. Pareto efficiency of LLOS guidance with current disturbance of $V_{c}=0.6 \mathrm{~m} / \mathrm{s}$ and $\beta_{c}=\pi / 4$. 


\section{VF Guidance}

The VF guidance law gives the desired heading as [7]:

$$
\psi_{d}=\alpha_{k}+\frac{2}{\pi} k_{v} \tan ^{-1}\left(k_{f} y_{e}\right),
$$

where $k_{v}, k_{f}>0$ are constant gains. Its path-following performance with and without current disturbance is shown in Figure 8, where the vehicle is also rendered to follow the path accurately under significant current disturbance. Its Pareto efficiency for the cases of disturbance and non-disturbance is shown in Figures 9 and 10.

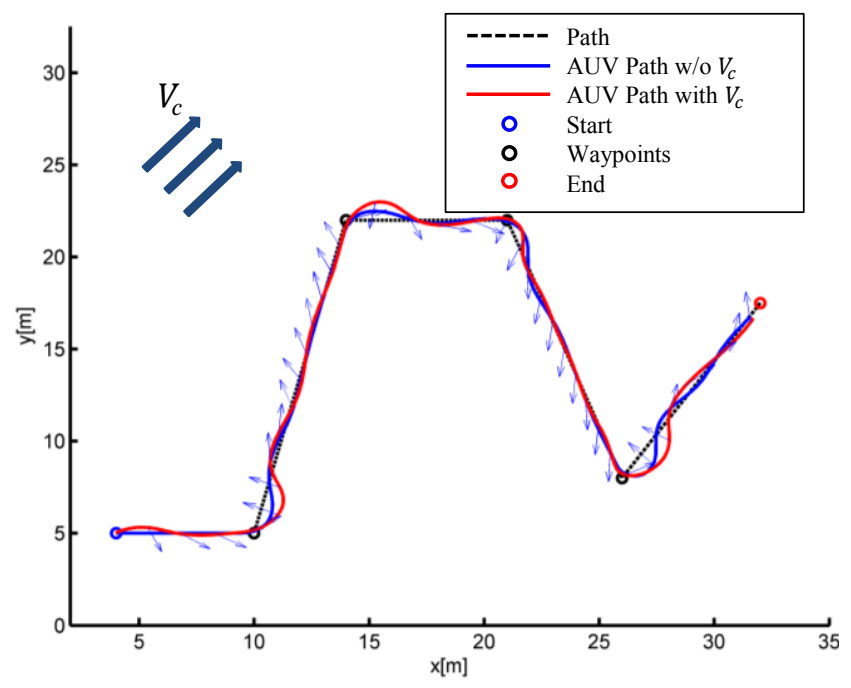

Fig. 8. Performance of VF guidance with $k_{v}=1.8, k_{f}=1.4$.

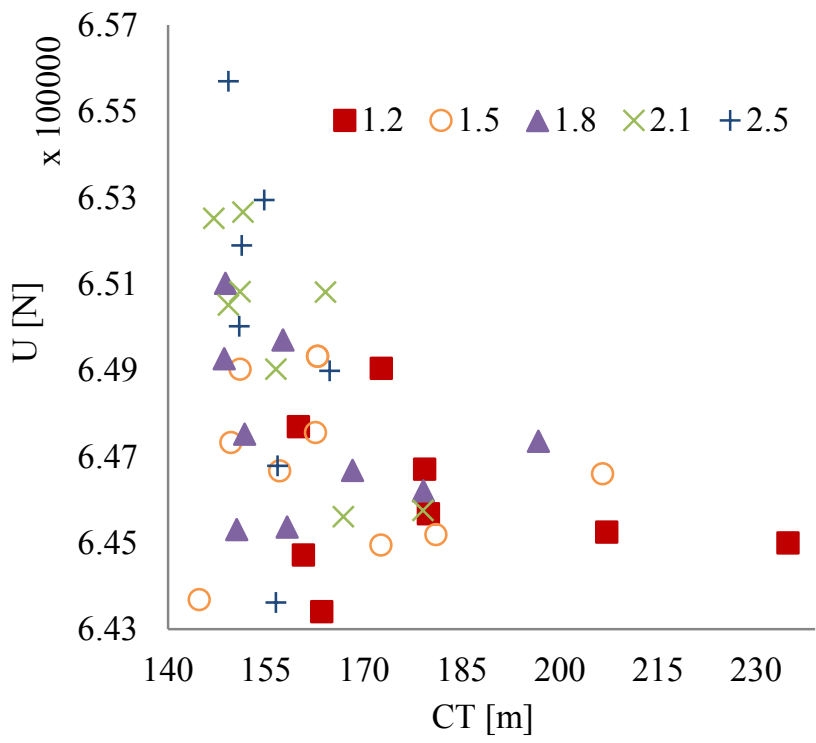

Fig. 9. Pareto efficiency of VF guidance without current disturbance.

\section{COMPARISON RESULTS}

The comparison is made using the tuning values that yield lowest cross-track error and control effort from the Pareto efficiency of both cases of disturbance and non-disturbance the corresponding guidance law. The cross-track error and control effort are shown in Table 1 for their best tuning values chosen from their corresponding Pareto efficiency plots. It can be seen that the LLOS guidance provides the lowest crosstrack error, but requires the highest control effort. VF guidance provides the lowest control effort, but with a crosstrack error higher than LLOS. The SELOS guidance gives the worst cross-tracking performance, but uses less control effort than LLOS. Therefore, there is no guidance law that is superior in both of the performance indexes. The summary of advantages and disadvantages of their performance are given in Table 2.

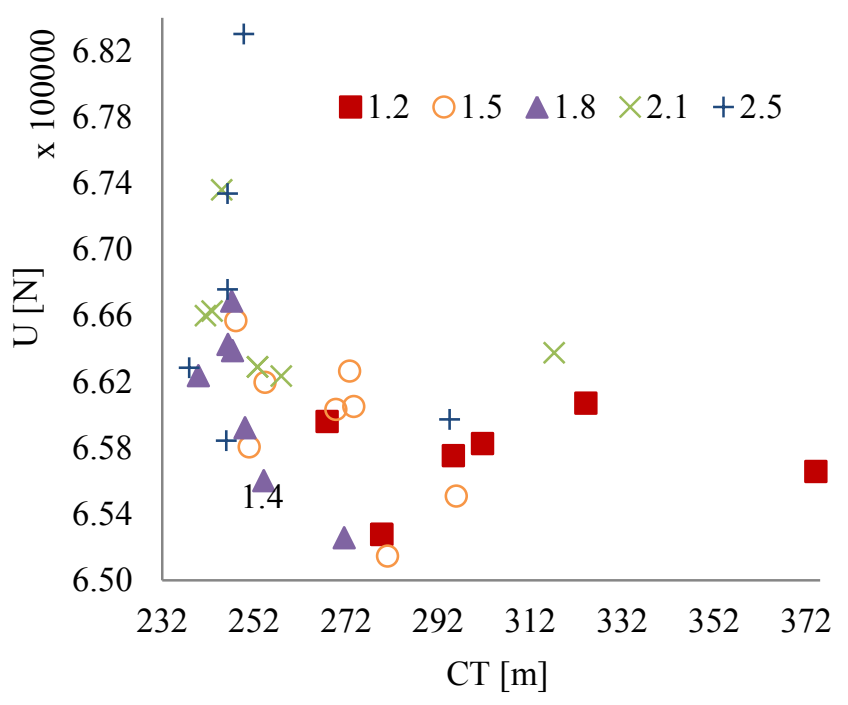

Fig. 10. Pareto efficiency of VF guidance with current disturbance of $V_{c}=$ $0.6 \mathrm{~m} / \mathrm{s}$ and $\beta_{c}=\pi / 4$.

\begin{tabular}{|c|c|c|c|}
\hline Guidance law & $\begin{array}{c}\text { Tuning } \\
\text { values }\end{array}$ & $\begin{array}{c}\text { Cross-track } \\
\text { error } \\
(\mathrm{CT}[\mathrm{m}])\end{array}$ & $\begin{array}{c}\text { Control } \\
\text { effort } \\
(\mathrm{U}[\mathrm{N}])\end{array}$ \\
\hline SELOS & $R_{\text {min }}=0.9$ & 169.9 & 646840 \\
\hline $\begin{array}{c}\text { SELOS } \\
\left(V_{c}=0.6 \mathrm{~m} / \mathrm{s}\right)\end{array}$ & & 267.5 & 658570 \\
\hline LLOS & & 149.9 & 648680 \\
\hline $\begin{array}{c}\text { LLOS } \\
\left(V_{c}=0.6 \mathrm{~m} / \mathrm{s}\right)\end{array}$ & $\Delta=0.4$ & 243.4 & 659490 \\
\hline VF & & 158.3 & 645360 \\
\hline $\begin{array}{c}\text { VF }\left(V_{c}=\right. \\
0.6 \mathrm{~m} / s)\end{array}$ & $k_{f}=1.4$ & 254.2 & 656020 \\
\hline
\end{tabular}

Table 1. The cross-track error and control effort produced for each scheme under the best tuning values. 


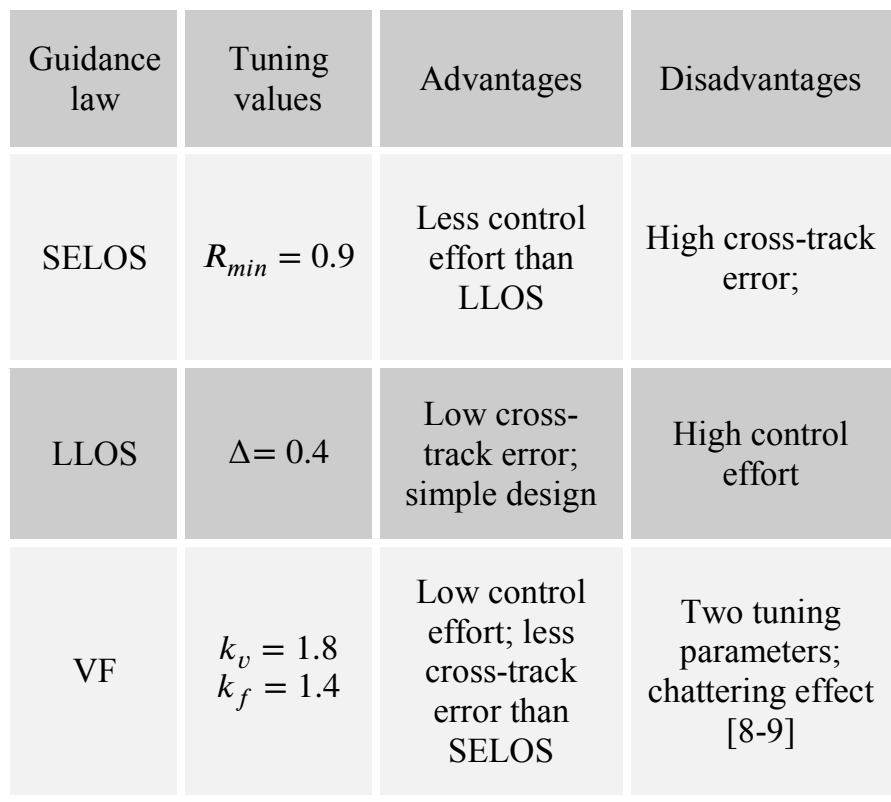

Table 2. Summary of the performance of the guidance laws.

\section{CONCLUSION}

The waypoint following performance and comparison of three popular guidance laws for AUVs are presented in this paper using the concept of relative kinematics for modelling ocean current disturbance. The SELOS guidance is an effective modification for the ELOS guidance that overcomes its drawback requiring additional path-approaching strategy for large cross-track errors. Performance of the guidance schemes are analyzed with and without significant current disturbances using the model of an AUV and a nonlinear feedback controller. The Pareto efficiency in terms of the total cross-track error and control as the performance index are used for the comparison, and multiple simulations are carried out to select the best tuning values for each guidance that results in low values in both performance categories. The results show that none of the guidance laws are the best in both categories, i.e. a guidance law that provides the lowest cross-track error does not provide the lowest control effort and vice versa. This implies that choice for selecting a best guidance law lies in the priorities of the application, i.e. whether a low cross-track error or a low control effort is more important for the requirements of the application. In addition, the number of tuning parameters and the chattering effect also have to be considered since there is no intuitive tuning methods if the VF guidance is chosen.

The comparison results will provide a good idea for users on how to select the best guidance for chosen an application involving AUVs, e.g. the VF guidance may be a good choice if energy efficiency is of utmost concern, and the LLOS guidance would be a good choice if cross-tracking is of high importance. If there are sufficient computational resources, a hybrid design can be an efficient approach where the vehicle can switch between the guidance laws, depending on the priorities of the task or the effects of environmental disturbances for instance.

\section{REFERENCES}

[1] B. Abdurahman, A. Savvaris, and A. Tsourdos, "A switching LOS guidance with relative kinematics for path-following of underactuated underwater vehicles", in Proc. $20^{\text {th }}$ IFAC World Congr., in press.

[2] A.L. Healey, and D. Lienard, "Multivariable sliding mode control for autonomous diving and steering control of autonomous underwater vehicles," IEEE J. Ocean Eng., vol. 18, Jul. 1993, pp. 327-339.

[3] E. Børhaug, A. Pavlov, and K.Y. Pettersen, "Integral LOS control for path following of underactuated marine surface vessels in the presence of constant ocean currents," in Proc. $47^{\text {th }}$ IEEE Conf. Decision and Control, Mexico, 2008, pp. 4984-4991.

[4] T.I. Fossen, M. Breivik, and R. Skjetne, "Line-of-sight path following of underactuated marine craft" in Proc. $6^{\text {th }}$ IFAC Conf. Maneuvering and Control of Marine Craft, Girona, 2003, pp. 244-249.

[5] T.I. Fossen, Handbook of marine craft hydrodynamics and motion control. Chichester, UK: John Wiley and Sons Ltd., 2011.

[6] M. Breivik, and T.I. Fossen, "Guidance laws for autonomous underwater vehicles," in Underwater Vehicles, A.V. Inzartsev, Ed. INTECK Education and Publishing, 2009, ch. 4, pp. 51-57.

[7] D.R. Nelson, D.B. Barber, T.W. McLain, and R.W. Beard, "Vector field path following of miniature air vehicles," IEEE Trans. Robotics, vol. 23, Jun. 2007, pp. 519-529.

[8] P.B. Sujit, S. Saripalli, and J.B. Sousa, "Unmanned aerial vehicle path following: a survey and analysis of algorithms for fixed-wing unmanned aerial vehicles," IEEE Trans. Control Systems Magazine, vol. 34, Feb. 2014, pp. 42-59.

[9] W. Caharija, K.Y. Pettersen, P. Calado, and J. Braga, "A comparison between the ILOS guidance and the vector field guidance," in Proc. $10^{\text {th }}$ IFAC Conf. Manoeuvring and Control of Marine Craft, Copenhagen, 2015, pp. 89-94.

[10] E. Fredriksen, and K.Y. Pettersen, "Global $\kappa$-exponential way-point manoeuvring of ships: theory and experiments," J. Automatica, vol. 42, 2006, pp. 677-687.

[11] G.C. Karras, C.P. Bechlioulis, M. Leonetti, N. Palomeras, P. Kormushev, K.J. Kyriakopoulos, and D.J. Caldwell, "On-line Identification of autonomous underwater vehicles through global derivative-free optimization," in Proc. IEEE/RSJ Int. Conf. Intelligent Robots and Systems, Tokyo, 2013, pp. 3859-3864. 